\title{
Quality of Life and Physical Ability Changes After Hospital-Based Cardiac Rehabilitation in Patients With Myocardial Infarction
}

\author{
Byung Joo Lee, $\mathrm{MD}^{1}$, Jin Young Go, $\mathrm{MD}^{1}$, Ae Ryung Kim, $\mathrm{MD}^{1}$, Seong Min Chun, $\mathrm{MD}^{2}$, \\ Minhyuk Park, $\mathrm{MS}^{3}$, Dong Heon Yang, $\mathrm{MD}^{4}$, Hun Sik Park, $\mathrm{MD}^{4}$, Tae-Du Jung, $\mathrm{MD}^{1,2}$ \\ ${ }^{1}$ Department of Rehabilitation Medicine, Kyungpook National University College of Medicine, Daegu; \\ ${ }^{2}$ Department of Rehabilitation Medicine, Kyungpook National University Medical Center, Daegu; \\ ${ }^{3}$ Department of Sports Medicine, Graduate School of Biomedical Science, Korea University, Sejong; \\ ${ }^{4}$ Department of Internal Medicine, Kyungpook National University Hospital, Daegu, Korea
}

\begin{abstract}
Objective To evaluate the effect of hospital-based cardiac rehabilitation (CR) on quality of life (QOL) and physical ability in patients with myocardial infarction (MI).

Methods Patients with MI who were referred to the Cardiac Health and Rehabilitation Center 2 weeks after percutaneous coronary intervention were divided into CR and non-CR groups. The CR group performed supervised exercises 3 times a week for 2 months. QOL assessment, using the 36-item Short-Form Health Survey (SF-36) and physical ability evaluation were performed at the beginning and end of CR.

Results The CR group demonstrated statistically significant improvements in physical functioning (PF), physical role functioning (RP), bodily pain (BP), general health perceptions (GH), vitality (VT), social role functioning (SF), emotional role functioning (RE), mental health (MH), physical component summary (PCS), and mental component summary (MCS). The non-CR group showed improvement in RP. Secondary outcomes, including resting heart rate (RHR), maximal oxygen consumption $\left(\mathrm{VO}_{2 \max }\right)$, metabolic equivalent of task (MET), maximal exercise time $\left(\mathrm{ET}_{\mathrm{max}}\right)$, stage 3 Borg rating of perceived exertion (3RPE), maximal Borg rating of perceived exertion (RPEmax), and stage 3 rate pressure product (3RPP), improved in the CR group. The non-CR group showed improvements in $\mathrm{VO}_{2 \max }, \mathrm{MET}, \mathrm{ET}_{\max }$, and 3RPE. There were significant differences in improvements in PF, RP, $\mathrm{BP}$, VT, SF, MH, MCS, RHR, $\mathrm{VO}_{2 \max }, \mathrm{MET}, \mathrm{ET}_{\max }, 3 \mathrm{RPE}$, and 3RPP between the two groups.

Conclusion Male patients with MI demonstrated improvements in QOL and physical ability following hospitalbased CR; the impact on the mental component was greater than that on the physical component.
\end{abstract}

Keywords Hospital-based home care, Cardiac rehabilitation, Myocardial infarction, Quality of life, Physical fitness

Received April 28, 2016; Accepted July 28, 2016

Corresponding author: Tae-Du Jung

Department of Rehabilitation Medicine, Kyungpook National University College of Medicine, 130 Dongdeok-ro, Jung-gu, Daegu 41944, Korea. Tel: +8253-200-5311, Fax: +82-53-423-0389, E-mail: teeed0522@hanmail.net

ORCID: Byung Joo Lee (http://orcid.org/0000-0002-6385-9256); Jin Young Go (http://orcid.org/0000-0002-6263-8355); Ae Ryung Kim (http://orcid. org/0000-0003-3765-3024); Seong Min Chun (http://orcid.org/0000-0002-2174-8742); Minhyuk Park (http://orcid.org/0000-0001-5204-5620); Dong Heon Yang (http://orcid.org/0000-0002-1646-6126); Hun Sik Park (http://orcid.org/0000-0001-7138-1494); Tae-Du Jung (http://orcid.org/0000-00021636-8665).

(a) This is an open-access article distributed under the terms of the Creative Commons Attribution Non-Commercial License (http://creativecommons.org/ licenses/by-nc/4.0) which permits unrestricted noncommercial use, distribution, and reproduction in any medium, provided the original work is properly cited. Copyright $\odot 2017$ by Korean Academy of Rehabilitation Medicine 


\section{INTRODUCTION}

The prevalence of myocardial infarction (MI) is very high, and its fatality rate is also high. Advances in medical management have contributed to a gradual decrease in the death rate for MI, but the prevalence of MI has increased.

Heart problems have a significant impact on patient quality of life (QOL), as the patients can become anxious, scared, and depressed. These emotional complications can limit the patient's activities of daily living, work performance, and relationships $[1,2]$. The magnitude of the impact of heart disease on an individual's QOL is due to the demanding long-term changes in lifestyle, coupled with the huge economic burden of treatment and rehabilitation [3].

Cardiac rehabilitation programs (CRPs), which were developed for the prevention and management of coronary artery disease (CAD), include exercise programs, nutrition consultation, smoking cessation education, medical treatment, weight management, CAD risk factor modification, and behavioral modification. They are also designed to improve QOL by improving the physical, emotional, and social functioning of each patient [4-7]. CRP consists of four phases. Phase I is the inpatient program. Phase II is the outpatient hospital-based program, during which we conducted our study, and phases III and IV are typically community-based programs. It is well known that CRPs can improve CAD risk factor management. It has also been reported that CRPs can help the patients to return to work and can improve their QOL $[8,9]$.

There have been many studies assessing the effects of CRPs. However, most of the studies focused on physical improvements [10-13]. More recently, as study designs have changed, QOL measures have been used to assess the treatment effects. The aim of this study was to investigate the effect of a phase II CRP on the QOL and physical abilities of patients with MI.

\section{MATERIALS AND METHODS}

\section{Subjects}

The study subjects were patients who were diagnosed with MI between June 2010 and June 2014 and referred to the Cardiac Health and Rehabilitation Center (CHRC) following percutaneous coronary intervention (PCI). The inclusion criteria for patients were as follows: (1) 2 weeks ( \pm 3 days) status post PCI, (2) age less than 70 years, (3) the Killip classification I, (4) ejection fraction $30 \%-50 \%$ on echocardiography, and (5) cognitive ability to complete the questionnaire.

The exclusion criteria were as follows: (1) hemodynamic instability (a decrease in continuous systolic blood pressure greater than $10 \mathrm{mmHg}$ or an increase in continuous systolic blood pressure to more than $250 \mathrm{mmHg}$ during exercise), (2) ST depression $>2 \mathrm{~mm}$, (3) uncontrolled cardiac arrhythmia with hemodynamic compromise, (4) congestive heart failure, or (5) inability to use the treadmill due to musculoskeletal pain.

The CRP was thoroughly explained to the study participants. Subjects who consented to participate in the CRP were included in the cardiac rehabilitation (CR) group. Subjects who agreed to participate in the regular followup but did not enroll in the hospital-based CRP were included in the non-CR group. Basic information, such as sex, age, education status, marital status, job status, and coexisting acute or chronic diseases, was documented for each group. To determine the presence of comorbid diseases, the patients completed questionnaires about hypertension, diabetes, cancer, tumor, stroke, osteoarthritis, rheumatic disease, chronic musculoskeletal pain, and dermatitis.

\section{Methods}

All of the participants underwent an exercise tolerance test (ETT) and completed a QOL questionnaire on their first visit to the CHRC, 2 weeks after they underwent a PCI. After the initial work-up, patients were thoroughly informed about the elements of the CRP and they made their decision regarding participation in CRP. After they made their decision regarding participation in CRP, both groups were educated on the recommended home-based exercises. The intensity of the home-based exercise program was based on the result of ETT in each patient. All participants were educated on their target heart rate, which was calculated such that it corresponded to $60 \%$ $80 \%$ of their maximal oxygen consumption. Participants were encouraged to reach their target heart rate while performing their home-based exercises. Patients in the non-CR group did not use a heart rate monitoring medical device. However, they were educated on self-monitoring of their heart rate by counting their radial pulse for 10 
seconds and multiplying that number by 6 . After the patients received thorough education on the recommended home-based exercise program, they were discharged. Over the following 2 months, the CR group participated in the hospital-based CRP. The non-CR group did not receive any specific rehabilitative treatment apart from selfadministered home-based exercises. After 2 months, the patients in both groups underwent another evaluation for comparison. Physical evaluation and questionnaires were administered by rehabilitation medicine doctors who were not involved in the study.

This study was conducted by Kyungpook National University Hospital and it was approved by the Institutional Review Board of Kyungpook National University (IRB No. 2016-03-004). Written informed consent was obtained from all participants in the study.

\section{Cardiac rehabilitation program}

The CR participants were taught by a physiatrist, CR therapist, nutritionist, and a coordinator during a 1-hour education session before they were discharged. The patients were provided with a published CHRC brochure on CAD during their initial education session and before discharge. Patient education included information about risk factor identification, a behavior change program, and counseling by a CR therapist. During the risk factor identification session, the physiatrist collected information on the patient's age, family history, and individual medical history including hypertension, diabetes, hypercholesterolemia, smoking, alcohol intake, obesity, and stress; the physiatrist then used this information to explain to each patient how these factors relate to CAD. Patients were educated on the intended effects and the potential side effects of their medications. During the behavior modification program, patients were educated on the methods to control their risk factors. In addition, the nutritionist performed counseling to help the participants improve their eating habits. Based on the basic information provided by the nutritionist, the rehabilitation coordinator explained to each patient the proper caloric intake and the recommended eating behavior. During the counseling session by the CR therapist, patients observed the exercises performed by other participants as well as they received a demonstration of the exercises from the therapist for a better understanding.

The CRP exercise prescriptions were based on the re- sults of the symptom-limited ETTs which the participants completed 2 weeks after they underwent PCI. The patients participated in the CRP for 60 minutes, 3 times a week for 8 weeks. The CRP consisted of 10-minute warmup exercises, 45-minute primary exercise program, and 5-minute cool-down exercises. The warm-up exercises included 2-minute walking and whole body stretching with the exercise therapists. The cool-down exercises also included whole body stretching. An electrocardiogram monitoring device was applied to each patient, during the entire exercise session. A single CR therapist monitored and supervised each patient in a 1-on-1 manner. After each session, proper feedback about the exercise performed was given to the patient by the CR therapist.

The 45-minute primary exercise component was composed of a repeated 15-minute long session that was further divided into 5-minute segments. The 15-minute session was repeated 3 times in total ( 45 minutes). One minute before each new segment, the patient's blood pressure, heart rate, and perceived exertion on the Borg rating of perceived exertion scale (RPE) [14] were measured to determine the exercise intensity for the next segment. The intensity was determined by the inclination and speed equivalent to one metabolic equivalent of task (MET) level. The program started with an intensity of $40 \%$ of Karvonen heart rate reserve (HRR) [15]. The goal for the first 4 weeks was $60 \%$ HRR, and the goal for the next 4 weeks was $80 \%$ HRR (Table 1 ).

\section{QOL assessment}

To determine the QOL of the patients in each group,

Table 1. Cardiac rehabilitation program

\begin{tabular}{ll}
\hline Mode & Aerobic \\
Method & Treadmill \\
Measure & BP, HR, RPE \\
Frequency, duration, & 3 day/wk, 60 min/day, \\
intensity & RPE 13-14 \\
Time to the goal (THR) & $40 \%$ HRR at the start \\
& $60 \%$ HRR in the first 4 weeks \\
& $80 \%$ HRR in the next 4 weeks \\
\hline
\end{tabular}

$\mathrm{BP}$, blood pressure; HR, heart rate; HRR, heart rate reserve; RPE, rate of perceived exertion; THR, target heart rate. $\mathrm{THR}=\left(\mathrm{HR}_{\max }-\mathrm{HR}_{\text {rest }}\right) \times(40 \%-80 \%)+\mathrm{HR}_{\text {rest }}$ $\mathrm{HRR}=\mathrm{HR}_{\max }-\mathrm{HR}_{\text {rest }}$ 
we used the second version of the 36-item Short-Form Health Survey (SF-36) [16]. The SF-36 consists of 36 questions in 8 categories: physical functioning (PF), physical role functioning (RP), bodily pain (BP), general health perceptions (GH), vitality (VT), social role functioning (SF), emotional role functioning (RE), and mental health (MH). Among the 8 categories, elements 1-4 create the physical component summary (PCS), and elements 5-8 create the mental component summary (MCS).

\section{Exercise ability assessment}

To determine physical functioning, the resting heart rate (RHR), maximal heart rate $\left(\mathrm{HR}_{\max }\right)$, maximal oxygen consumption $\left(\mathrm{VO}_{2 \max }\right)$, MET, maximal exercise time $\left(\mathrm{ET}_{\max }\right)$, maximal perceived exertion rating $\left(\mathrm{RPE}_{\max }\right)$, stage 3 rating of perceived exertion (3RPE), maximal rate pressure product $\left(R P P_{\text {max }}\right)$, stage 3 rate pressure product (3RPP), and respiratory exchange ratio (RER) were measured during the ETT. The ETT was performed using the modified Bruce protocol, which is divided into successive 3minute stages, each of which requires the patient to walk faster and at a steeper grade. To prevent bias on the part of the therapist, the results of the initial ETT were not provided to the designated therapist during the reassessment period.

\section{Statistical analysis}

Statistical analyses were performed using SPSS ver.
18.0 (SPSS Inc., Chicago, IL, USA). A paired t-test was performed to assess improvements in QOL and physical ability in each group. Comparisons between the CR group and non-CR group were performed using an independent $\mathrm{t}$-test. The $\mathrm{p}$-values less than 0.05 were considered statistically significant.

\section{RESULTS}

Basic information about the CR group and non-CR group From June 2010 to June 2014, 353 MI patients were referred to the CHRC. Out of the referred patients, 230 patients consented to participate in the study. Patients who consented to participate in the CRP were included in the CR group $(\mathrm{n}=139)$. The remaining patients participated in the regular follow-up without undergoing the CRP $(\mathrm{n}=91)$. In the CR group, 67 participants dropped out due to a variety of reasons; while in the non-CR group, 41 patients dropped out. Therefore, a total of 122 patients were included in the analysis (Fig. 1). There was no restriction on gender in the inclusion criteria; however, all participants were male. The only health-related problems that developed during therapy were pain due to herniation of the nucleus pulposus in the cervical spine $(n=1)$ and pain due to knee osteoarthritis $(n=1)$.

The mean age of the enrolled patients was 54.8 years, and all of them were married men. Age, sex, marital status, education status, job status, and comorbid disease

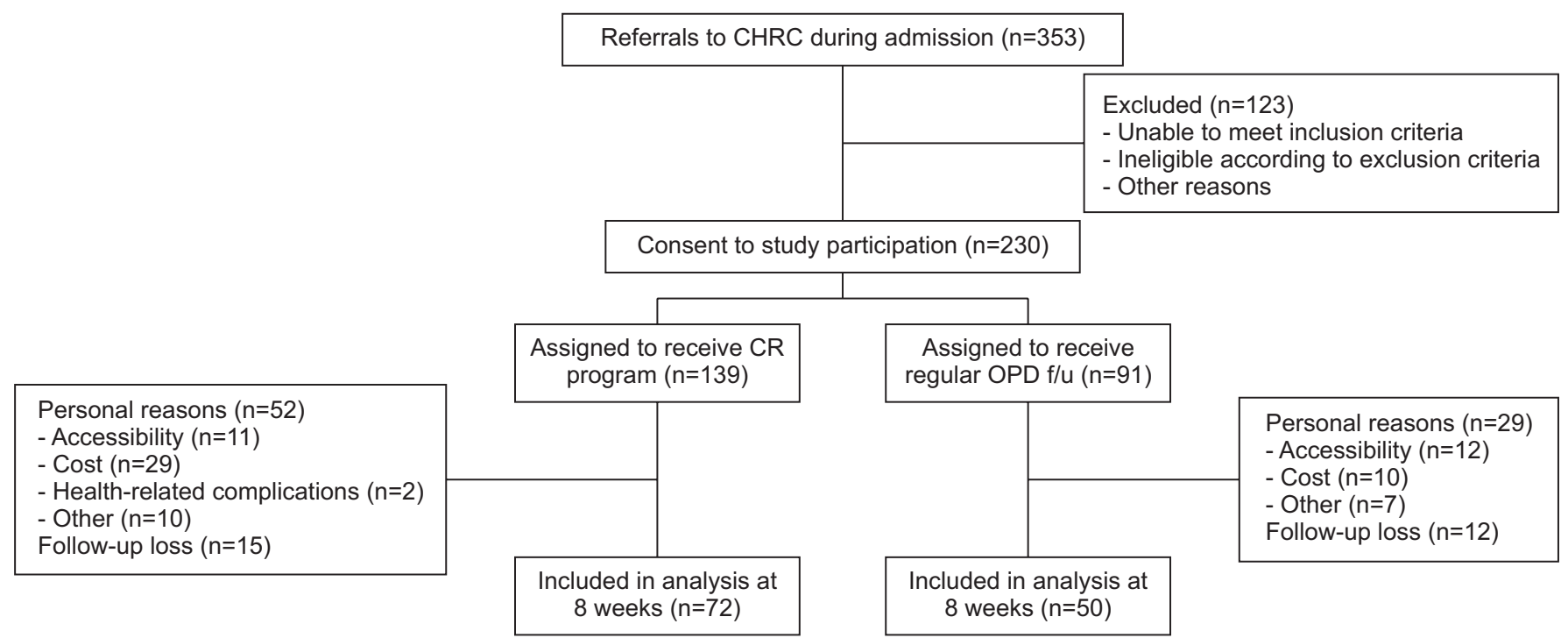

Fig. 1. Flow of participants through the study. CHRC, the Cardiac Health and Rehabilitation Center; CR, cardiac rehabilitation; OPD, outpatient department; f/u, follow-up. 
are all known to impact QOL. Our analysis showed that these variables were similar in both groups. The demographic data of patients are presented in Table 2.

\section{OOL changes after the CRP and comparison between} the $C R$ and non-CR groups

The SF-36 results for the CR group revealed improvements in every category. The PF, RP, BP, GH, VT, SF, RE, MH, PCS, and MCS showed statistically significant improvements $(\mathrm{p}<0.05)$. The SF-36 results for the non-CR group revealed a statistically significant improvement in RP $(\mathrm{p}<0.05)$. Comparative analysis of SF-36 QOL scores between the CR group and non-CR group showed statistically significant differences in PF, RP, BP, VT, SF, MH, and MCS (Table 3). There were no significant differences in the baseline parameters between the two groups.

Physical ability improvement after the CRP and comparison between the $\mathrm{CR}$ and non-CR groups

The RHR, $\mathrm{VO}_{2 \max }, \mathrm{MET}, \mathrm{ET}_{\max }$, 3RPE, $\mathrm{RPE}_{\max }$, and 3RPP demonstrated significant changes $(p<0.05)$ in the $C R$

Table 2. Comparison of socio-demographic variables and chronic diseases between the CR group and the non-CR group

\begin{tabular}{lccc}
\hline \multicolumn{1}{c}{ Variable } & $\begin{array}{c}\text { CR } \\
\text { group } \\
\text { (n=72) }\end{array}$ & $\begin{array}{c}\text { Non-CR } \\
\text { group } \\
(\mathbf{n = 5 0 )}\end{array}$ & p-value \\
\hline Age (yr) & $54.8 \pm 8.3$ & $54.3 \pm 7.7$ & 0.882 \\
Sex, male & 100 & 100 & 1.000 \\
Family status, married & 100 & 100 & 1.000 \\
Education & & & 0.795 \\
$\quad$ Middle school & 10.0 & 16.7 & \\
High school & 45.0 & 40.0 & \\
\hline University & 45.0 & 43.3 & \\
Work outdoors & 80.0 & 63.3 & 0.365 \\
Chronic co-morbidity & & & \\
\hline Diabetes mellitus & 20.0 & 20.0 & 1.000 \\
\hline Hypertension & 15.0 & 23.3 & 0.720 \\
\hline Malignancy & 10.0 & 6.7 & 0.670 \\
\hline Stroke & 0.0 & 0.0 & 1.000 \\
\hline Musculoskeletal problem & 5.0 & 6.7 & 0.808 \\
\hline Skin disease & 5.0 & 10.0 & 0.523 \\
\hline
\end{tabular}

Values are presented as mean \pm standard deviation or percentage.

$\mathrm{CR}$, cardiac rehabilitation. group. $\mathrm{HR}_{\max }(\mathrm{p}=0.194), \mathrm{RPP}_{\max }(\mathrm{p}=0.285)$, and RER $(\mathrm{p}=$ 0.192 ) did not exhibit relevant changes. The non-CR group showed statistically significant improvements in $\mathrm{VO}_{2 \max }$, MET, $\mathrm{ET}_{\max }$, and 3RPE $(\mathrm{p}<0.05)$. The CR group showed significantly greater improvements in RHR, $\mathrm{VO}_{2 \max }$, MET, $\mathrm{ET}_{\text {max }}, 3 \mathrm{RPE}$, and 3RPP than the non-CR group (Table 4). There were no significant differences in the baseline parameters between the two groups.

\section{DISCUSSION}

In this study, we determined that participation in a hospital-based CRP improved both the physical and mental aspects of the QOL of patients after MI. Although a few improvements in select categories were noted in the nonCR group, the comparative analysis revealed remarkable improvements in the CR group. The enhancements in the physical ability categories were consistent with the improvements noted in the physical aspect-related questions of the SF-36. The most significant finding of our study was that the mental aspects of QOL improved after participating in the CRP, and not only the physical aspects. The combined improvements in these two health aspects resulted in overall enhancement of quality of life.

We evaluated the effects of a hospital-based CRP on the QOL of patients with MI. After participation in the CRP, we observed statistically significant improvements in every category of the SF-36. Our findings correspond with the results of many earlier studies that demonstrated improvements in the MCS, PCS, and all the subdomains of the SF-36. Khalife-Zadeh et al. [17] reported that patient awareness of the principles of rehabilitation and gradual resumption of activities improved both physical and mental health indices. Duarte et al. [18] reported regarding the importance of a comprehensive CRP owing to its positive impact on physical health, anxiety, depression, and sleep quality. Aude et al. [19] reported that CR helps improve patient perceptions of QOL, which enhances both the mental and physical components of QOL.

Another important finding of our study was that the mental component of QOL improved more than the physical component. A key factor that could explain our result was the exercise environment. During the CRP, all patients were under the strict supervision of an individual CR therapist. Therefore, the patients were likely to feel emotionally stable and be free from the fear of a recurrent 
Table 3. Comparative analysis of SF-36 quality of life scores

\begin{tabular}{|c|c|c|c|c|c|}
\hline Category & Group & Baseline & After 8 weeks & p-value $e^{\text {a) }}$ & p-value ${ }^{\text {b) }}$ \\
\hline \multirow[t]{2}{*}{ Physical functioning } & Non-CR & $89.67 \pm 8.55$ & $90.00 \pm 8.66$ & 0.705 & $0.043^{*}$ \\
\hline & CR & $86.25 \pm 13.24$ & $91.96 \pm 7.37$ & $0.002^{*}$ & \\
\hline \multirow[t]{2}{*}{ Physical role functioning } & Non-CR & $56.67 \pm 37.16$ & $36.67 \pm 22.89$ & $0.045^{*}$ & $0.011^{*}$ \\
\hline & CR & $68.75 \pm 38.26$ & $84.11 \pm 29.47$ & $0.013^{*}$ & \\
\hline \multirow[t]{2}{*}{ Bodily pain } & Non-CR & $80.53 \pm 19.29$ & $78.93 \pm 17.20$ & 0.592 & $0.041^{*}$ \\
\hline & CR & $77.18 \pm 20.80$ & $87.75 \pm 13.56$ & $0.005^{*}$ & \\
\hline \multirow[t]{2}{*}{ General health perceptions } & Non-CR & $63.40 \pm 18.92$ & $66.60 \pm 16.84$ & 0.314 & 0.194 \\
\hline & $\mathrm{CR}$ & $59.39 \pm 19.40$ & $65.96 \pm 16.15$ & $0.024^{*}$ & \\
\hline \multirow[t]{2}{*}{ Vitality } & Non-CR & $56.33 \pm 19.41$ & $56.67 \pm 21.52$ & 0.717 & $0.037^{*}$ \\
\hline & CR & $57.02 \pm 17.39$ & $64.02 \pm 14.50$ & $0.001^{*}$ & \\
\hline \multirow[t]{2}{*}{ Social role functioning } & Non-CR & $76.50 \pm 21.19$ & $81.83 \pm 17.07$ & 0.075 & $0.046^{*}$ \\
\hline & $\mathrm{CR}$ & $65.78 \pm 26.70$ & $81.25 \pm 18.48$ & $<0.001^{*}$ & \\
\hline \multirow[t]{2}{*}{ Motional role functioning } & Non-CR & $55.56 \pm 48.25$ & $84.45 \pm 35.34$ & 0.086 & 0.902 \\
\hline & $\mathrm{CR}$ & $56.64 \pm 40.98$ & $82.14 \pm 31.46$ & $0.004^{*}$ & \\
\hline \multirow[t]{2}{*}{ Mental health } & Non-CR & $66.40 \pm 19.41$ & $68.67 \pm 17.43$ & 0.539 & $0.044^{*}$ \\
\hline & $\mathrm{CR}$ & $59.08 \pm 18.59$ & $71.25 \pm 16.37$ & $<0.001^{*}$ & \\
\hline \multirow[t]{2}{*}{ Physical component summary } & Non-CR & $49.35 \pm 4.54$ & $51.31 \pm 5.22$ & 0.078 & 0.139 \\
\hline & CR & $50.25 \pm 7.15$ & $52.39 \pm 3.94$ & $0.025^{*}$ & \\
\hline \multirow[t]{2}{*}{ Mental component summary } & Non-CR & $40.09 \pm 11.22$ & $42.77 \pm 8.51$ & 0.397 & $0.048^{*}$ \\
\hline & $\mathrm{CR}$ & $38.12 \pm 12.23$ & $46.16 \pm 8.78$ & $<0.001^{*}$ & \\
\hline
\end{tabular}

Values are presented as mean \pm standard deviation.

$\mathrm{CR}$, cardiac rehabilitation.

${ }^{a)} \mathrm{p}$-value in a single group by paired t-test. ${ }^{\text {b) }} \mathrm{p}$-value between the two groups by independent $\mathrm{t}$-test.

${ }^{*} \mathrm{p}<0.05$.

cardiac crisis. This emotional stability motivated the CRP participants to adhere to the rehabilitation process more strictly than the non-CR participants [20]. In addition to the emotional support that the participants received, the constant real-time feedback from the monitor and the therapist made the therapy much more efficient and effective. The non-CR participants would have exercised more efficiently if they had been able to use a portable monitoring device that could provide real-time feedback on their exercise performance.

The physical ability parameters improved in both groups. Brannon et al. [21] reported that the effects of CR were primarily due to increased efficiency in peripheral tissue oxygen consumption, which consequently increases $\mathrm{VO}_{2 \max }$, rather than the central effect on the heart. Scheuer and Tipton [22] and Laughlin et al. [23] reported more efficient metabolism and increased oxygen extraction in striated muscle after aerobic exercise. Due to the effects of aerobic exercise, blood flow requirements at the same exercise intensity decrease over time. Blood pressure and myocardial oxygen consumption decrease, and the heart rate decreases at rest and during submaximal exercise due to an increase in vagal nerve stimulation. In our study, the improvements in the physical ability parameters may be explained by these effects. The fact that the CR group exhibited improvements in more parameters than the non-CR group indicates that the CRP was performed effectively.

This study has some limitations. The first limitation is that this was a retrospective study. Therefore, the patients who had a less favorable attitude towards the CRP were not enrolled in our study. In addition, factors, such as socio-economic status, employment status, or education level which could have had an influence on the study result were not assessed. Also, selection bias could have potentially affected our results, due to the fact that all our study participants were male. To overcome these disadvantages, a prospective study is needed. Second, the 
Table 4. Comparative analysis of physical ability parameters

\begin{tabular}{|c|c|c|c|c|c|}
\hline Category & Group & Baseline & After 8 weeks & p-value ${ }^{\text {a) }}$ & p-value ${ }^{\text {b) }}$ \\
\hline \multirow[t]{2}{*}{ RHR } & Non-CR & $63.13 \pm 9.51$ & $64.47 \pm 11.83$ & 0.753 & $0.005^{*}$ \\
\hline & $\mathrm{CR}$ & $64.50 \pm 9.15$ & $56.75 \pm 7.18$ & $<0.001^{*}$ & \\
\hline \multirow[t]{2}{*}{$\mathrm{HR}_{\max }$} & Non-CR & $148.13 \pm 17.24$ & $152.40 \pm 16.95$ & 0.155 & 0.429 \\
\hline & $\mathrm{CR}$ & $148.50 \pm 18.24$ & $150.71 \pm 15.24$ & 0.194 & \\
\hline \multirow[t]{2}{*}{$\mathrm{VO}_{2 \max }$} & Non-CR & $29.02 \pm 5.62$ & $30.15 \pm 5.88$ & $0.029^{*}$ & $0.047^{*}$ \\
\hline & $\mathrm{CR}$ & $29.43 \pm 3.90$ & $32.83 \pm 4.47$ & $<0.001^{*}$ & \\
\hline \multirow[t]{2}{*}{ MET } & Non-CR & $8.29 \pm 1.61$ & $8.61 \pm 1.68$ & $0.023^{*}$ & $0.026^{*}$ \\
\hline & $\mathrm{CR}$ & $8.40 \pm 1.12$ & $9.45 \pm 1.20$ & $<0.001^{*}$ & \\
\hline \multirow[t]{2}{*}{$\mathrm{ET}_{\max }$} & Non-CR & $899.40 \pm 93.56$ & $1,005.73 \pm 288.02$ & $0.011^{*}$ & $0.003^{*}$ \\
\hline & $\mathrm{CR}$ & $926.29 \pm 88.25$ & $1041.39 \pm 69.82$ & $<0.001^{*}$ & \\
\hline \multirow[t]{2}{*}{ 3RPE } & Non-CR & $10.13 \pm 2.48$ & $8.47 \pm 1.89$ & $0.017^{*}$ & $0.029^{*}$ \\
\hline & $\mathrm{CR}$ & $11.32 \pm 2.14$ & $8.11 \pm 1.50$ & $<0.001^{*}$ & \\
\hline \multirow[t]{2}{*}{$\mathrm{RPE}_{\max }$} & Non-CR & $16.40 \pm 1.96$ & $16.00 \pm 2.36$ & 0.531 & 0.452 \\
\hline & $\mathrm{CR}$ & $16.96 \pm 1.77$ & $16.00 \pm 1.68$ & $0.011^{*}$ & \\
\hline \multirow[t]{2}{*}{ 3RPP } & Non-CR & $12,788.47 \pm 2,788.20$ & $12,886.20 \pm 3,128.30$ & 0.865 & $0.002^{*}$ \\
\hline & $\mathrm{CR}$ & $13,140.04 \pm 2,878.40$ & $10,425.64 \pm 1,450.75$ & $<0.001^{*}$ & \\
\hline \multirow[t]{2}{*}{$\mathrm{RPP}_{\max }$} & Non-CR & $22,989.27 \pm 3,015.66$ & $25,301.27 \pm 3,754.24$ & 0.053 & 0.491 \\
\hline & $\mathrm{CR}$ & $24,438.25 \pm 5,307.52$ & $25,627.96 \pm 4,778.78$ & 0.285 & \\
\hline \multirow[t]{2}{*}{ RER } & Non-CR & $1.26 \pm 0.20$ & $1.22 \pm 0.08$ & 0.932 & 0.452 \\
\hline & CR & $1.14 \pm 0.10$ & $1.17 \pm 0.11$ & 0.192 & \\
\hline
\end{tabular}

Values are presented as mean \pm standard deviation.

$\mathrm{RHR}$, resting heart rate; $\mathrm{HR}_{\max }$, maximal heart rate; $\mathrm{VO}_{2 \max }$, maximal oxygen consumption; MET, metabolic equivalent of task; $\mathrm{ET}_{\max }$, maximal exercise time; $3 \mathrm{RPE}$, rating of perceived exertion at stage 3; $\mathrm{RPE}_{\max }$, maximal rating of perceived exertion; 3RPP, rate pressure product at stage 3 ; $\mathrm{RPP}_{\max }$, maximal rate pressure product; RER, respiratory exchange ratio; CR, cardiac rehabilitation.

${ }^{a)} \mathrm{p}$-value in a single group by paired t-test. ${ }^{\text {b) }} \mathrm{p}$-value between the two groups by independent $\mathrm{t}$-test. ${ }^{*} \mathrm{p}<0.05$.

drop-out rate among the participants was high, which makes it difficult to generalize the results of this study. Given the retrospective study design, a lower drop-out rate would have strengthened the results of the study. Lack of self-awareness with regard to the magnitude of the disease and the cost of the treatment program appeared to be the main reasons for the high patient dropout rate. Additional studies that explore the reasons why patients drop out of CRPs would be helpful to identify strategies to reduce the drop-out rates. Third, comparative analyses of the patient's age and severity of disease were not performed. Fourth, disease severity of the participants was mild due to the strict inclusion criteria. Therefore, our results may not represent the experience of all patients after MI and PCI.

In conclusion, participation in an 8-week hospital- based CRP for male patients with MI resulted not only in improvements in physical abilities, but also in QOL. In addition, the mental component of the QOL assessment revealed greater improvements than the physical component. Therefore, a hospital-based CRP should be offered to all eligible patients with MI.

\section{CONFLICT OF INTEREST}

No potential conflict of interest relevant to this article was reported.

\section{REFERENCES}

1. Heo S, Lennie TA, Okoli C, Moser DK. Quality of life in patients with heart failure: ask the patients. Heart 


\section{Lung 2009;38:100-8.}

2. Law MR, Watt HC, Wald NJ. The underlying risk of death after myocardial infarction in the absence of treatment. Arch Intern Med 2002;162:2405-10.

3. World Health Organization. Prevention of cardiovascular disease: guidelines for assessment and management of cardiovascular risk. Geneva: World Health Organization; 2007.

4. Skodova Z, van Dijk JP, Nagyova I, Rosenberger J, Ondusova D, Middel B, et al. Psychosocial predictors of change in quality of life in patients after coronary interventions. Heart Lung 2011;40:331-9.

5. Frattaroli J, Weidner G, Merritt-Worden TA, Frenda $\mathrm{S}$, Ornish D. Angina pectoris and atherosclerotic risk factors in the multisite cardiac lifestyle intervention program. Am J Cardiol 2008;101:911-8.

6. Leon AS, Franklin BA, Costa F, Balady GJ, Berra KA, Stewart KJ, et al. Cardiac rehabilitation and secondary prevention of coronary heart disease: an American Heart Association scientific statement from the Council on Clinical Cardiology (Subcommittee on Exercise, Cardiac Rehabilitation, and Prevention) and the Council on Nutrition, Physical Activity, and Metabolism (Subcommittee on Physical Activity), in collaboration with the American association of Cardiovascular and Pulmonary Rehabilitation. Circulation 2005;111:369-76.

7. Savage PD, Sanderson BK, Brown TM, Berra K, Ades PA. Clinical research in cardiac rehabilitation and secondary prevention: looking back and moving forward. J Cardiopulm Rehabil Prev 2011;31:333-41.

8. Anderson L, Thompson DR, Oldridge N, Zwisler AD, Rees K, Martin N, et al. Exercise-based cardiac rehabilitation for coronary heart disease. Cochrane Database Syst Rev 2016;1:CD001800.

9. Clark AM, Hartling L, Vandermeer B, McAlister FA. Meta-analysis: secondary prevention programs for patients with coronary artery disease. Ann Intern Med 2005;143:659-72.

10. Dunagan J, Adams J, Cheng D, Barton S, Bigej-Cerqua J, Mims L, et al. Development and evaluation of a treadmill-based exercise tolerance test in cardiac rehabilitation. Proc (Bayl Univ Med Cent) 2013;26:247-51.

11. Kim C, Youn JE, Choi HE. The effect of a self exercise program in cardiac rehabilitation for patients with coronary artery disease. Ann Rehabil Med 2011;35:381-7.
12. Ribeiro F, Oliveira NL, Silva G, Campos L, Miranda F, Teixeira M, et al. Exercise-based cardiac rehabilitation increases daily physical activity of patients following myocardial infarction: subanalysis of two randomised controlled trials. Physiotherapy 2015 Dec 25 [Epub]. http://dx.doi.org/10.1016/j.physio.2015.12.002

13. Szot W, Zajac J, Kubinyi A, Kostkiewicz M. The effects of cardiac rehabilitation on overall physical capacity and myocardial perfusion in women with microvascular angina. Kardiol Pol 2016;74:431-8.

14. Borg GA. Psychophysical bases of perceived exertion. Med Sci Sports Exerc 1982;14:377-81.

15. Karvonen J, Vuorimaa T. Heart rate and exercise intensity during sports activities. Practical application. Sports Med 1988;5:303-11.

16. Han CW, Lee EJ, Iwaya T, Kataoka H, Kohzuki M. Development of the Korean version of Short-Form 36Item Health Survey: health related QOL of healthy elderly people and elderly patients in Korea. Tohoku J Exp Med 2004;203:189-94.

17. Khalife-Zadeh A, Dorri S, Shafiee S. The effect of cardiac rehabilitation on quality of life in patients with acute coronary syndrome. Iran J Nurs Midwifery Res 2015;20:588-93.

18. Duarte Freitas P, Haida A, Bousquet M, Richard L, Mauriege P, Guiraud T. Short-term impact of a 4-week intensive cardiac rehabilitation program on quality of life and anxiety-depression. Ann Phys Rehabil Med 2011;54:132-43.

19. Aude T, Hill PD, Anderson MA. Quality of life after participation in a rural phase II cardiac rehabilitation program. J Nurs Care Qual 2006;21:56-62.

20. Pietrabissa G, Ceccarini M, Borrello M, Manzoni GM, Titon A, Nibbio F, et al. Enhancing behavioral change with motivational interviewing: a case study in a Cardiac Rehabilitation Unit. Front Psychol 2015;6:298.

21. Brannon FJ, Foley MW, Star JA, Saul LM. Cardiopulmonary rehabilitation: basic theory and application. 3rd ed. Philadelphia: F. A. Davis; 1998. Chapter 4, Physiologic adaptations to aerobic exercise; pp. 70-83.

22. Scheuer J, Tipton CM. Cardiovascular adaptations to physical training. Annu Rev Physiol 1977;39:221-51.

23. Laughlin MH, Davis MJ, Secher NH, van Lieshout JJ, Arce-Esquivel AA, Simmons GH, et al. Peripheral circulation. Compr Physiol 2012;2:321-447. 